\title{
STRUKTUR UND HERKUNFT DER WILDBIENEN-POPULATIONEN AUF FELDERN DER ZOTTELWICKE IN UNGARN. EIN VERGLEICH MIT POPULATIONEN IN ANDEREN GEBIETEN EUROPAS UND IN ANDEREN FUTTERLEGUMINOSEN 1
}

\author{
Structure et origine des populations d'abeilles sauvages \\ dans les champs de vesce velue en Hongrie \\ Comparaison avec des populations d'autres régions d'Europe \\ et d'autres légumineuses fourragères
}

\author{
Paul BENEDEK
}

Prognose-Zentrum im Zentralen Pflanzenschutz- und Quarantäne-Laboratorium, Ministerium für Landwirtschaft und Ernährung, Budapest/Ungarn ${ }^{2}$

\begin{abstract}
SUMMARY
STRUCTURE AND ORIGIN OF HAIRY VETCH POLLINATING WILD BEE POPULATIONS IN HUNGARY AND THEIR RELATIONSHIPS TO POPULATIONS

IN OTHER TERRITORIES OF EUROPE AND IN OTHER FODDER LEGUMES

Based on the results of four years' extensive surveys definite differences are demonstrated in the structure of hairy vetch pollinating wild bee populations in certain regions of Hungary. Due to the overwhelming density of Bombus species the structure of the populations in the North-western regions is related to that of North and West European populations. In contrast, in the Great Hungarian Plain - Central and South-eastern Hungary - the density of the Eucera, Tetralonia and Andrena species is striking. This is explained by the Mediterranian and Pontic origin of the hairy vetch pollinating wild bee populations in the Hungarian Great Plain. Several ecological conditions indicate a definite primary or secondary connection. between certain medium flight wild bees — Eucera and Tetralonia species — and winter vetches. It is concluded that in Southern Europe the structure of the hairy vetch pollinating
\end{abstract}

1. Ưbersetzung aus dem Englischen F. RutrNer, Oberursel.

2. H-1502 Budapest, Budaörsi ut 141, Postfach 127. 
wild bee populations may be closely similar as in the Great Hungarian Plain. Several hairy vetch pollinating wild bee species are important in the pollination of lucerne and red clover, therefore, these cultivars are mutually of a definite influence on the density of their pollinating wild bee populations.

\section{ZUSAMMENFASSUNG}

Ausgehend von den Ergebnissen vierjähriger ausgedehnter Untersuchungen werden eindeutige Unterschiede in der Zusammensetzung der Populationen von Wildbienen nachgewiesen, die in bestimmten Gebieten Ungarns die Zottelwicke bestäuben. Wegen der übergrossen Häufigkeit von Hummelarten zeigen die Populationen in den nordwestlichen Regionen des Landes Ähnlichkeiten mit den Populationen von Nord- und Westeuropa. Im Gegensatz dazu überwiegen in der Ungarischen Tiefebene die Arten der Gattungen Eucera, Tetralonia und Andrena. Das ist mit der mediterranen und pontischen Herkunft dieser Arten zu erklären. Es bestehen einige deutliche ökologische Hinweise auf primäre oder sekundäre Beziehungen zwischen Wildbienen mittlerer Flugzeit (Eucera- und Tetralonia-Arten) und Winterwicken. Daraus kann der Schluss gezogen werden, dass die Struktur der Wildbienen-Populationen auf der Zottelwicke in Südeuropa und in der Ungarischen Tiefebene ziemlich ähnlich ist. Einige Bestäuber der Zottelwicke unter den Wildbienen sind auch für die Bestäubung der Luzerne und des Rotklees von Bedeutung. Daher beein flussen diese Kulturen ihrerseits die Populationsdichte ihrer Bestäuber in erheblichem Ausmass.

\section{EINLEITUNG}

Die Zottelwicke (Vicia villosa Roth.) ist selbststeril (Schelhorn, 1942, 1946) und deshalb auf Insektenbestäubung angewiesen. Infolge der typischen zygomorphen Blütenstruktur kann die Bestäubung nur von Bienen (Hymenoptera : Apoidea) durchgeführt werden. Neben Honigbienen wurden auf den Feldern der Zottelwicke in Europa vor allem Hummelarten festgestellt (Schelhorn, 1942, 1946; Ruszkowski und Bilinski, 1969). Dass auch andere Wildbienenarten auftreten, wurde erst in letzter Zeit berichtet (BENEDEK et al., 1971).

Im Gegensatz dazu wurden in den Feldern der Zottelwicke in den U.S.A. Wildbienen aus mehreren Gattungen beobachtet (BoharT, 1960). Die Autoren beschränkten sich jedoch auf die Zusammenstellung von Listen der bestäubenden Arten oder die Feststellung ihrer Dominanz in den Kulturen. Daher gibt es noch keine Informationen über die Dichte und die Herkunft der bestäubenden Wildbienen in den Feldern der Zottelwicke. Das hat uns veranlasst, in den Jahren 1968-1971 eingehende Untersuchungen über die WildbienenPopulationen in mehreren Feldern der Zottelwicke durchzuführen.

\section{MATERIAL UND METHODE}

Die Untersuchungen wurden in den Komitaten Békés und Hajdu in den Jahren 1968-1971 durchgeführt, im Komitat Györ 1969-1971 und in den Komitaten Szolnok und Vas in den Jahren 1970 und 1971 (Tabelle 1). Die Beobachtungen wurden in Feldern von 30-40 ha durchgeführt, die in voller Blüte standen und die in verschiedenen Gebieten der untersuchten Komitate nach dem Zufall ausgewählt worden waren. Auf diese Weise wurden Daten von. ziemlich gleichmässiger regionaler Verteilung gewonnen. 


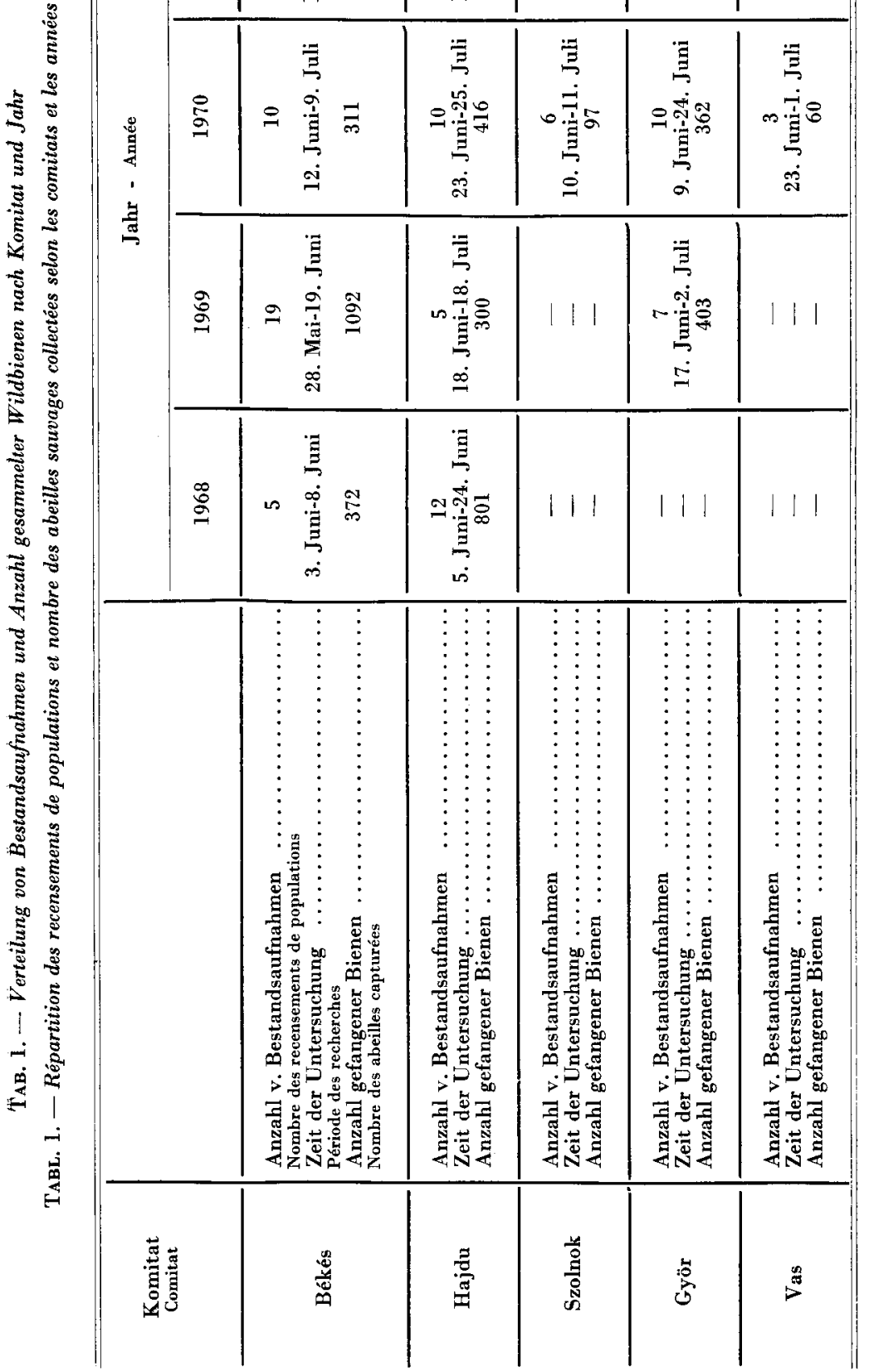


Die Beobachtungen wurden teils in der Ungarischen Tiefebene (Komitat Békés, Hajdu und Szolnok) mit trockenwarmem Klima und in dem kühleren und feuchten Nordwesten des Landes (Komitate Györ und Vas) gewonnen. Diese zwei Gebiete sind durch das Ungarische Mittelgebirge voneinander getrennt (Abbildung 1).

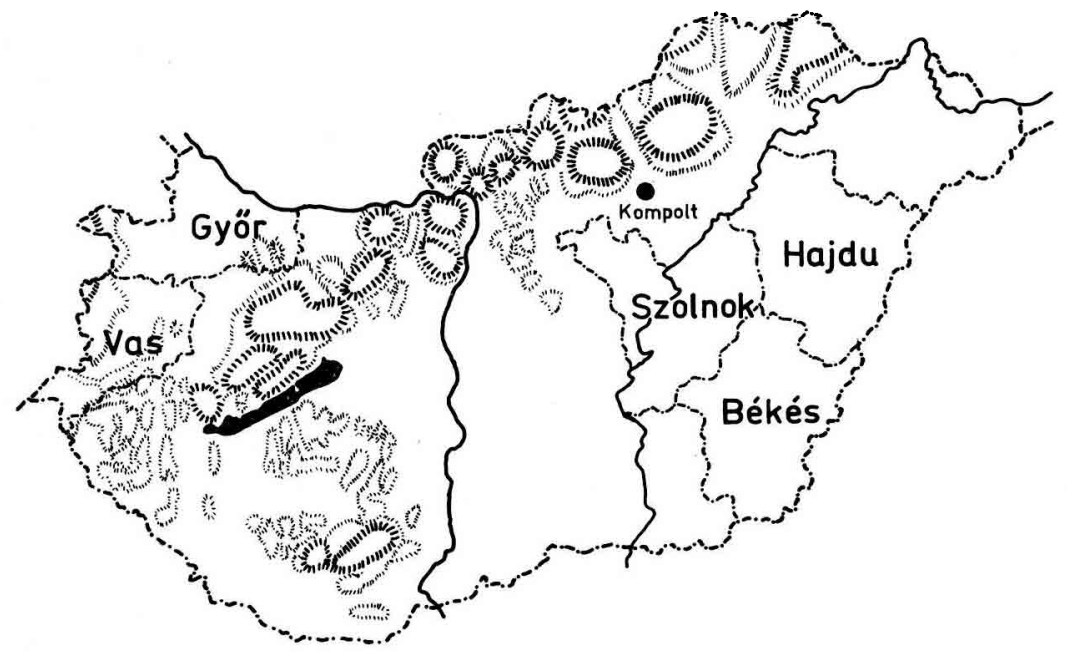

Aвв. 1. - Verteilung der untersuchten Gebiete im Nordwesten Ungarns (Komitate Györ und Vas) und in der Ungarischen Tiefebene (Komitate Békés, Hajdu und Szolnok). Bergrücken, Hügel, der Balaton See und die Flüsse Donau und Teiss sind ebenfalls dargestellt.

FIG. 1 - Répartition des régions étudiées dans le nord-ouest de la Hongrie (comitats de Györ et de Vas, et dans la plaine hongroise (comitats de Bëkés, Hajdu et Szolnok). Les montagnes, les collines, le lac Balaton le Danube et la Teiss sont également représentés.

Dichte und spezifische Zusammensetzung der Wildbienen-Populationen wurden nach der “ einfachen Streifenmethode " des Autors bestimmt. Dabei geht man mit einer konstanten Geschwindigkeit von $10 \mathrm{~m}$ pro Minute durch das Feld, den Blick unter einem Winkel von $45^{\circ}$ auf die Blütenköpfe gerichtet, und fängt mit einem Insektennetz von $30 \mathrm{~cm}$ Durchmesser in einem $0,50 \mathrm{~m}$ breiten Streifen alle Wildbienen. Bei einer Länge des Streifens von 600 oder $300 \mathrm{~m}$ ergibt das eine Fläche von $300 \mathrm{bzw} .150 \mathrm{~m}^{2}$. Zur genauen Erfassung der Populationsdichte wurden auch all die Bienen registriert, die mit dem Netz nicht gefangen werden konnten. Die reichlich vorhandenen Honigbienen wurden in den meisten Fällen zwar ebenfalls registriert, auf sie wird aber in dieser auf die Wildbienen beschränkten Untersuchung nicht weiter eingegangen (die Bestäubungstätigkeit der Honigbiene wurde von WeAvER (1954-1965) eingehend untersucht). Theoretisch ergibt diese Methode dieselben Resultate wie eine gleichzeitige Registrierung der Population in einem bestimmten Augenblick (BenedeK 1970 a). Deshalb kann die Populationsdichte in dem beobachteten Streifen durch Addition der Zahlen der gefangenen und der registrierten Wildbienen bestimmt werden. Anschliessend wurde aus den Fängen die spezifische Zusammensetzung der Population festgestellt.

Insgesamt wurden 120 Streifenaufnahmen durchgeführt und dabei 5095 Wildbienen gefangen (Tabelle 1). Ausserdem wurden im Bereich der Streifen 425 Individuen notiert, aber nicht gefangen. Nur $9,4 \%$ der Tiere waren Männchen.

Schon früher wurde gezeigt, dass in Populationen von Wildbienen ganz bestimmte Typen der Flugdauer festgestellt werden können (BENEDEK 1968). Diese bestehen aus Arten, die sich primär oder sekundär an entomophile Elemente einer bestimmten Vegetationsphase anpassen. Deshalb wurden sowohl die Dichte (D) wie die Konstanz des Auftretens (C) von Flugtypen, bzw. Arten der Wildbienen analysiert. Die Dichte wurde aus der Gesamtzahl gefangener und beobachteter Individuen pro Aufnahme errechnet. $\mathrm{Zu}$ jedem Mittelwert wird der mittlere Fehler angegeben, in der " Konstanz " kommt die Zahl der Aufnahmen zum Ausdruck, in denen eine bestimmte Bienenart beobachtet wurde. Die mittlere Dichte und die Werte für die Konstanz einiger Arten sind in Tabelle 2 wiedergegeben. Die mittlere Dichte von Flugtypen ist in den Abbildungen 3 und 4, angegeben. 


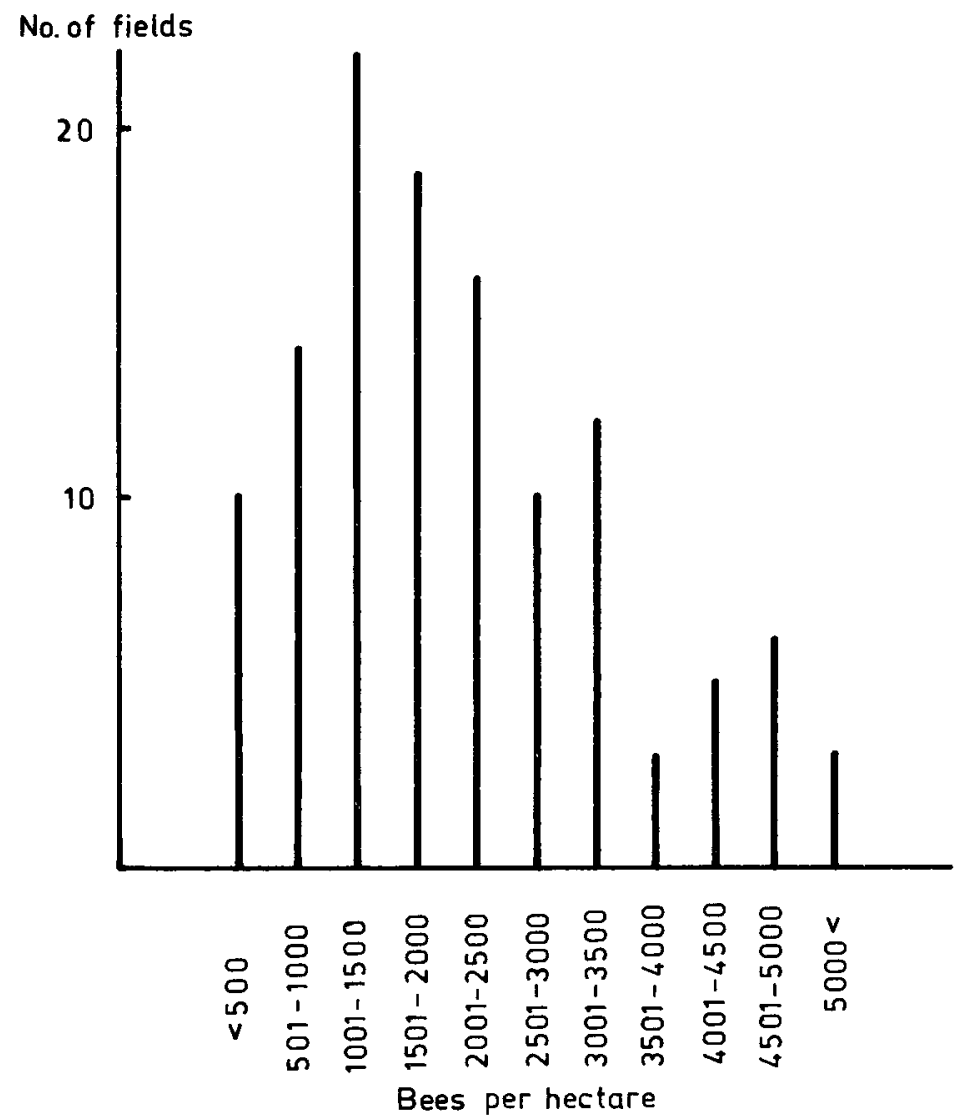

Авв. 2. - Anzahl der durchgeführten Bestandsaufnahmen in blühenden Feldern der Zottelwicke in Bezug auf die Dichte der Populationen bestäubender Wildbienen.

Fıg. 2. - Nombre des recensements de populations effectués dans les champs de vesce velue en fleurs en relation avec la densité des populations d'abeilles sauvages pollinisatrices. Verticalement : nombre de champs; horizontalement : nombre d'abeilles par hectare. 


\begin{tabular}{|c|c|c|c|c|c|c|}
\hline \multirow{7}{*}{ 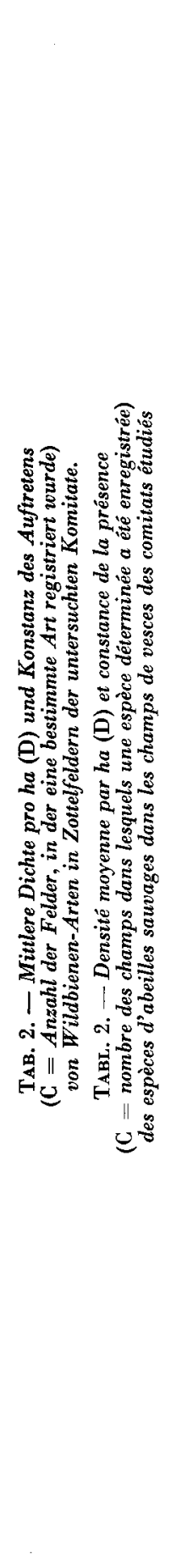 } & \multirow{2}{*}{ 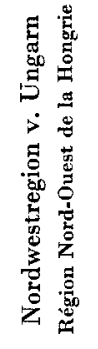 } & 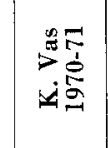 & $\begin{array}{l}0 \\
\theta\end{array}$ & $\begin{array}{l}11 \\
11\end{array}$ & $\begin{array}{l}|1||1| \\
|1| 1 \mid 1\end{array}$ & 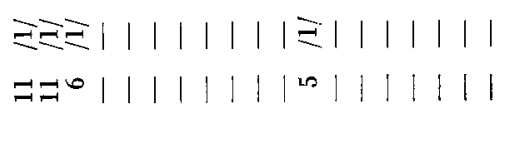 \\
\hline & & 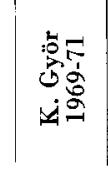 & $\begin{array}{l}0 \\
\theta\end{array}$ & $\begin{array}{l}11 \\
11\end{array}$ & $\begin{array}{l}|||||| \\
||||||\end{array}$ & 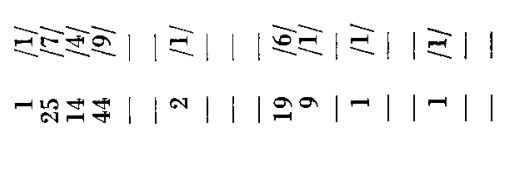 \\
\hline & \multirow{3}{*}{ 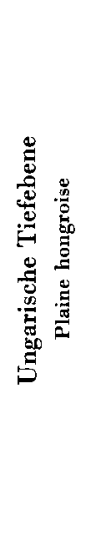 } & 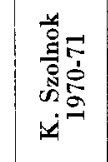 & $\begin{array}{l}0 \\
0\end{array}$ & $\begin{array}{l}11 \\
11\end{array}$ & $\begin{array}{l}|1| 1 \mid \\
|1| 1 \mid\end{array}$ & 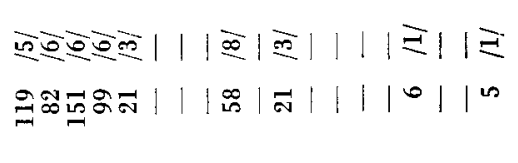 \\
\hline & & 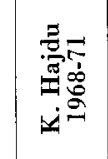 & 0 & 러 & 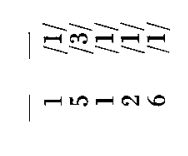 & 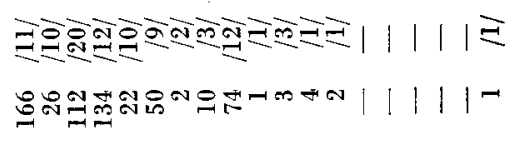 \\
\hline & & 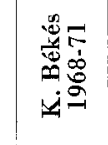 & $\begin{array}{l}n \\
\theta\end{array}$ & & 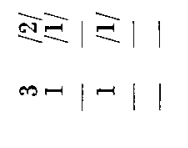 & 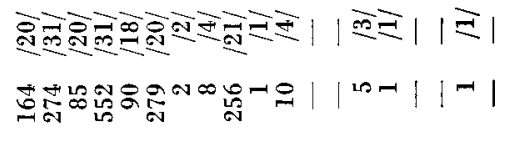 \\
\hline & & 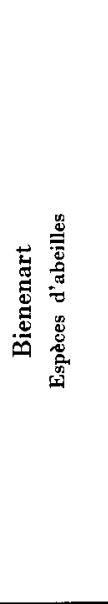 & & 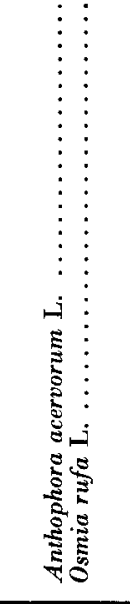 & 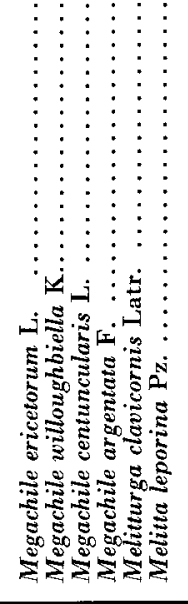 & 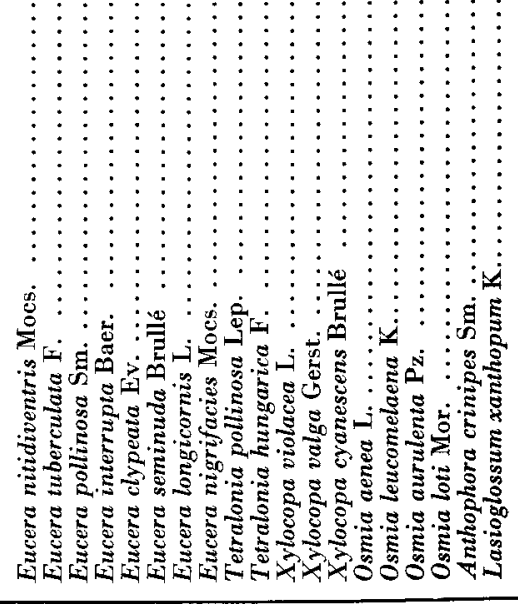 \\
\hline & & 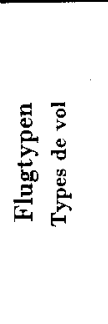 & & 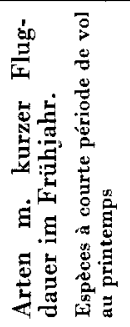 & 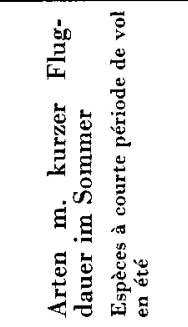 & 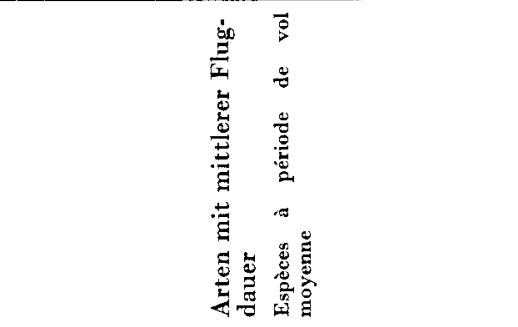 \\
\hline
\end{tabular}




\begin{tabular}{|c|c|c|}
\hline 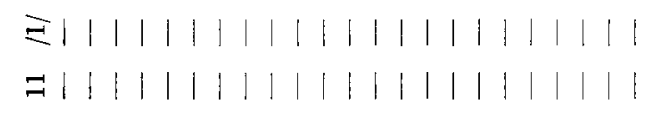 & 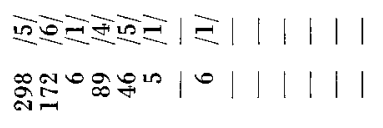 & \\
\hline 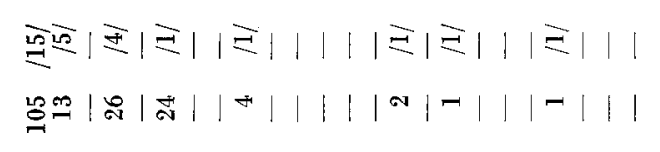 & 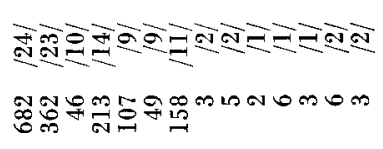 & ล \\
\hline 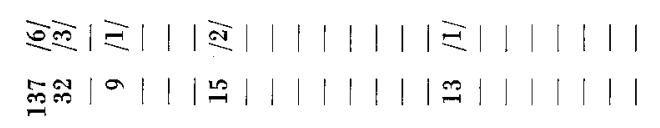 & 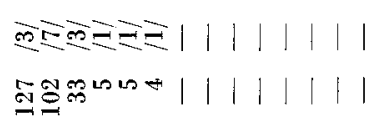 & $\Rightarrow$ \\
\hline 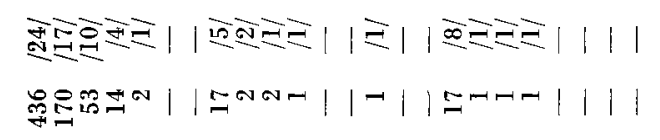 & 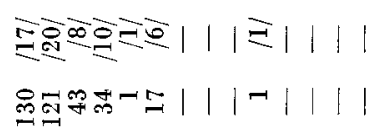 & ఉे \\
\hline 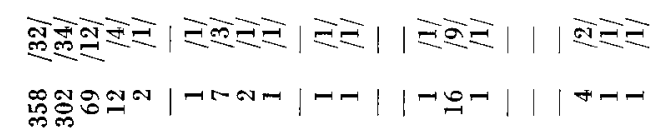 & 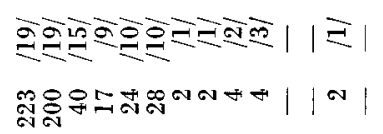 & $\mathscr{2}$ \\
\hline 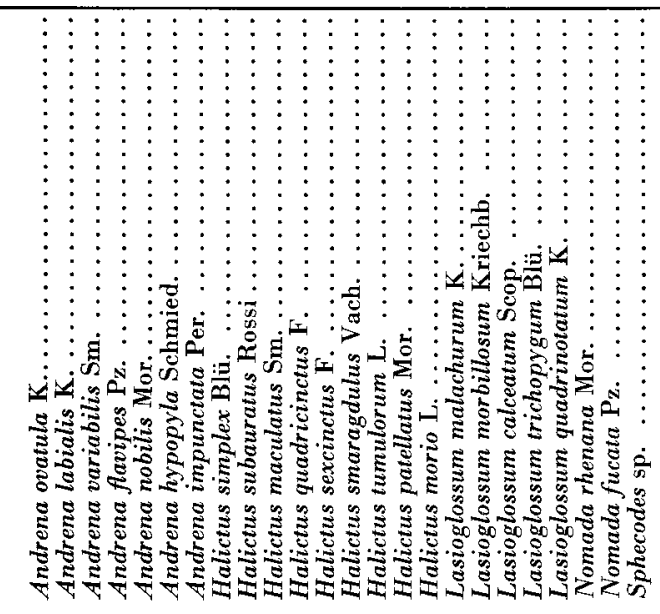 & 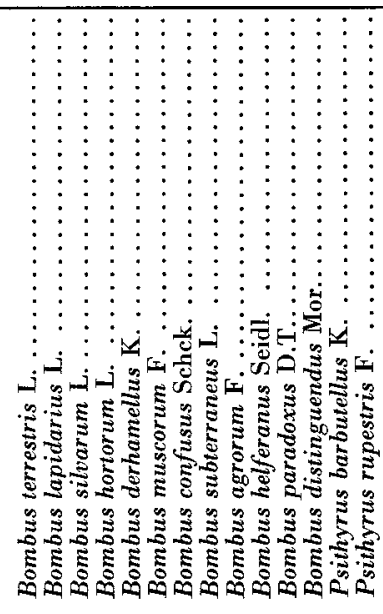 & 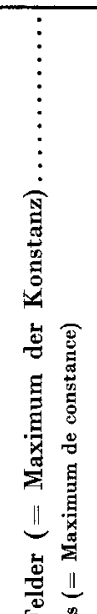 \\
\hline 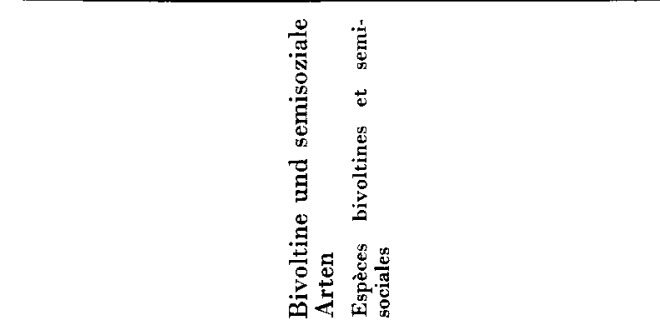 & 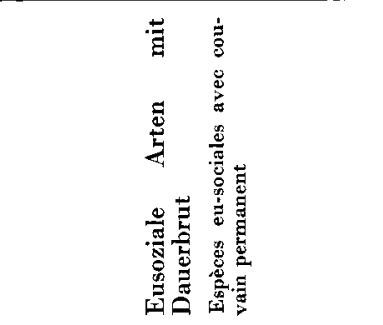 & 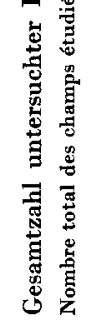 \\
\hline
\end{tabular}




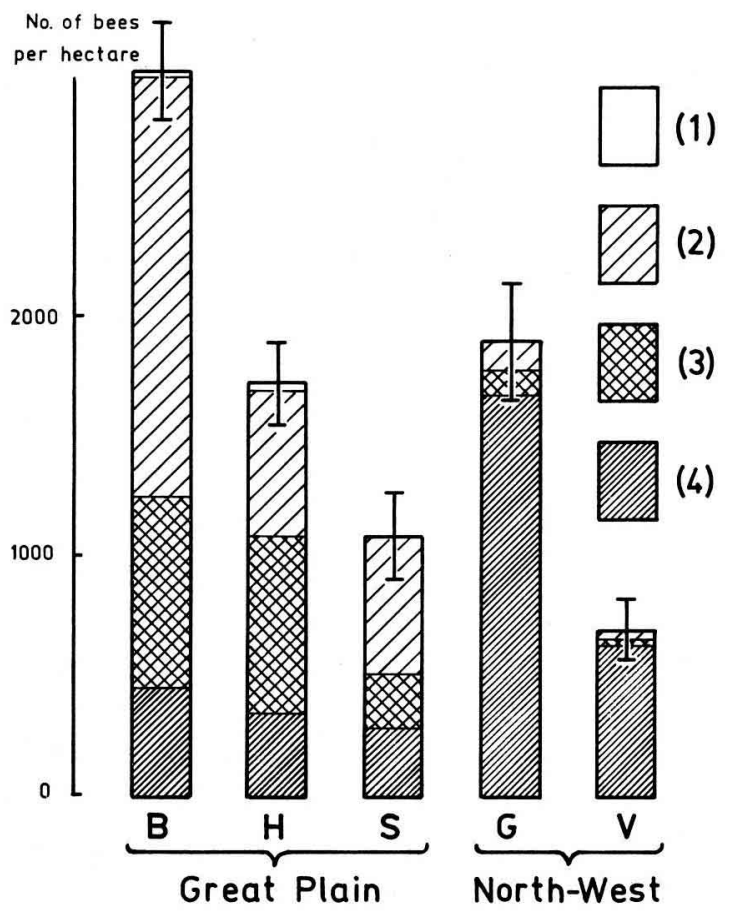

Abb. 3. - Mittlere Populationsdichte (mit Standardabweichungen) von Wildbienen und der Anteil der Flugtypen in der Ungarischen Tiefebene und im Nordwesten Ungarns.

1. Arten mit kurzer Flugdauer im Frühjahr und Sommer.

2. Arten mit mittlerer Flugdauer.

3. Bivoltine (= zwei Generationen in einem Sommer) und semisoziale Arten.

4. Dauerbrütende eusoziale Arten. Komitate $: \mathrm{B}=$ Békés. $\mathrm{H}=$ Hajdu, $\mathrm{S}=$ Szolnok, $\mathrm{V}=$ Vas.

Fig. 3. - Densité moyenne de populations (avec écart-type) d'abeilles sauvages et répartition des types de vol dans la plaine hongroise et le nord-ouest de la Hongrie.

1. Espèces à courte durée de vol au printemps et en été.

2. Espèces à durée de vol moyenne.

3. Espèces bivoltines (deux générations au cours d'un été) et espèces semi-sociales.

4. Espèces eu-sociales à couvain permanent Comitats : $\mathrm{B}=$ Békés, $\mathbf{H}=\mathrm{Hajdu}, \mathrm{S}=\mathrm{Szolnok}, \mathrm{V}=\mathrm{Vas}$. 


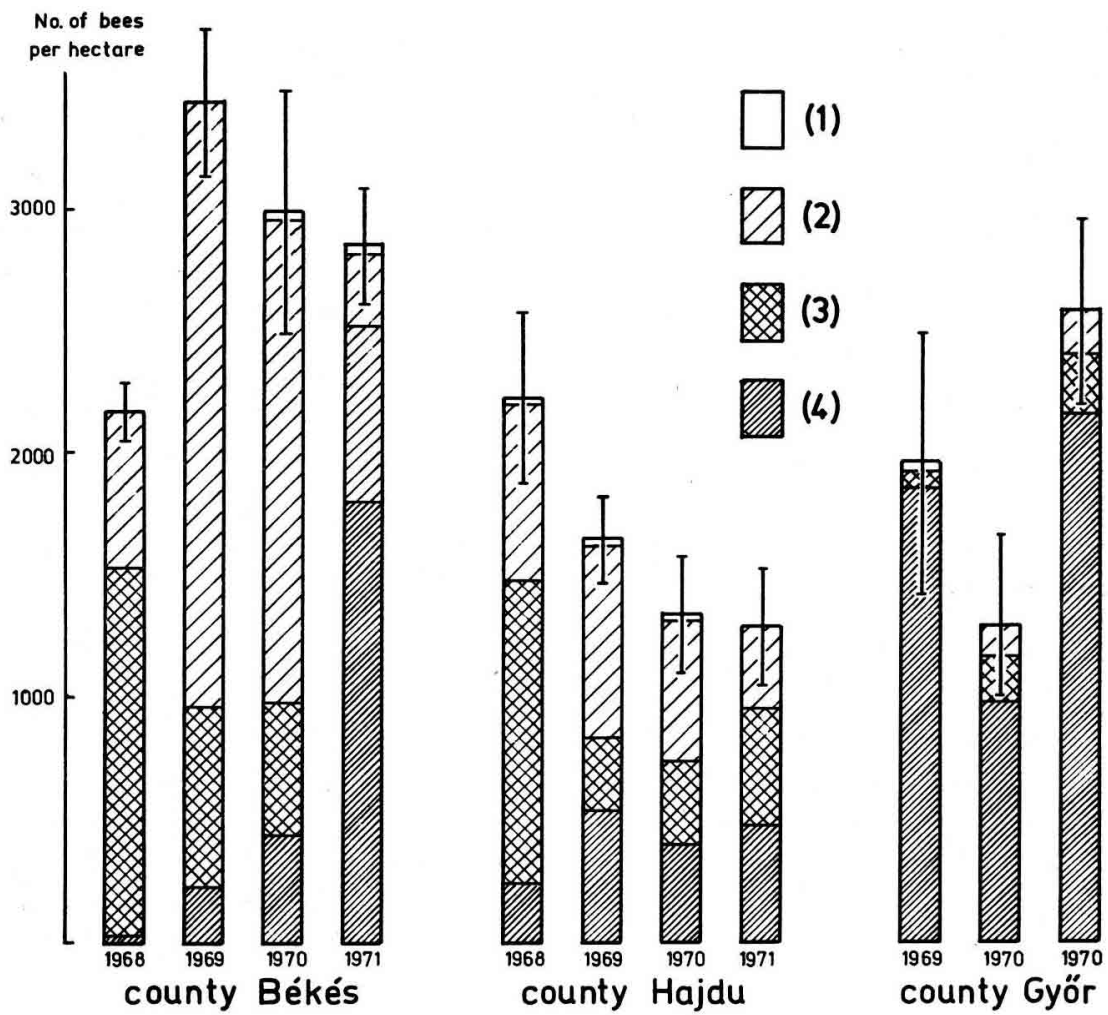

Abb. 4. - Mitulere Populationsdichte (mit Standardabweichungen) von Wildbienen und der Anteil der Flugtypen in den Komitaten Békés, Hajdu und Györ in aufeinanderfolgenden Jahren. (Erklärung wie in Abbildung 3.)

FIG. 4. - Densités moyennes de populations (avec écart-type) d'abeilles sauvages et répartition des types de vol dans les comitats de Bêkês, Hajdn Györ dans des années successives. (Mêmes conventions graphiques que figure 3.) 


\section{RESULTATE}

Die Dichte der Wildbienen schwankte auf den untersuchten Feldern zwischen 300-5600 pro Hektar (Abbildung 2). Die mittlere Dichte betrug $2117 \pm 121$ pro ha. Die mittlere Wildbienendichte zeigte aber beträchtliche Unterschiede nach Komitaten (Abbildung 3) und Jahren (Abbildung 4). In den blühenden Feldern der Zottelwicke wurden 65 Arten von Wildbienen festgestellt, die in fünf Typen der Flugzeit (Fluggruppen) auftraten (Tabelle 2).

Von den Wildbienen des Frühjahrs und Sommers mit kurzer Flugzeit wurden nur wenige Arten registriert (Tabelle 2). Ihr Vorkommen konnte nur auf wenigen Feldern der Ungarischen Tiefebene in geringer Dichte beobachtet werden.

Arten mit mittlerer Flugdauer hingegen traten in beachtlicher Anzahl auf (Tabelle 2). Unter ihnen übertreffen einige Eucera-Arten (E. interrupta, E. tuberculata, E. pollinosa, E. clypeata, E. seminuda) und Tetralonia pollinosa alle übrigen an Dichte und Konstanz. Die Bienen mit mittlerer Flugdauer waren in der Ungarischen Tiefebene in grosser Zahl vorhanden, während sie im Nordwesten nur mit geringer Dichte und Konstanz auftraten (Tabelle 2, Abbildung 3).

Die Zahl der nachgewiesenen Arten von bivoltinen und semisozialen Bienen mit langer Flugzeit übertrifft die der anderen Flugtypen (Tabelle 2). Ihre Dichte zeigt in den verschiedenen Komitaten grosse Unterschiede (Abbildung 3). Die Dichte von zwei Arten (Andrena ovatula, A. labialis) war beträchtlich und in einigen der untersuchten Komitate zeigte auch eine dritte Art (A. variabilis) eine beachtliche Häufigkeit. Im Gegensatz dazu zeigten die restlichen Arten nur geringe Dichten und auch ihre Konstanz war unbedeutend, mit Ausnahme von Halictus maculatus und Lassioglossum malachurum (Tabelle 2).

Auch von den kontinuierlich brütenden eusozialen Wildbienen mit langer Flugzeit wurden einige Arten festgestellt (Tabelle 2). Einige von ihnen (Bombus terrestris, $B$. lapidarius) traten in besonders grosser Dichte auf, andere Arten wie Bombus hortorum, B. silvarum und B. muscorum zeigten zwar eine beachtliche Dichte, sie traten aber nur mit geringer Konstanz auf (Tabelle 2). Die restlichen Arten erwiesen sich lediglich als gelegentliche Besucher in den Feldern der Zottelwicke und sie wurden deshalb nur bei einigen wenigen Aufnahmen registriert.

Deutliche Unterschiede konnten sowohl hinsichtlich des Anteils der Flugtypen und der Dichte ihrer Arten festgestellt werden. In der Ungarischen Tiefebene stammt der Hauptteil der Populationen aus den Gruppen der Wildbienen mit mittlerer und langer Flugzeit, mit zwei Generationen in einer 
Saison (bivoltin), während im Nordwesten die kontinuierlich brütenden Hummeln den weitaus grössten Anteil stellten (Abbildung 3).

Obwohl innerhalb dieser beiden Regionen gewisse Unterschiede in der Struktur und Dichte der Bienenpopulation gefunden wurden, waren diese doch gering im Vergleich zu den Unterschieden zwischen diesen Regionen.

Die Daten von drei Komitaten (Békés, Haidu, Györ) reichten aus, um die jährliche Fluktuation in der Hauptzusammensetzung der Populationen zu zeigen (Abbildung 4). Es kann nachgewiesen werden, dass die grossen Unterschiede zwischen der Ungarischen Tiefebene (Komitat Békés und Hajdu) und dem Nordwesten (Komitat Györ) trotz der beträchtlichen jährlichen Schwankungen in der Struktur und Dichte der Populationen konstant blieben.

\section{DISKUSSION UND SCHLUSSFOLGERUNGEN}

Die Zusammensetzung der als Bestäuber tätigen Wildbienen-Populationen in Feldern der Zottelwicke zeigt beim Vergleich verschiedener Regionen Ungarns sehr auffallende Unterschiede. Wegen des starken Überwiegens der Hummelarten gleicht der feuchte und kühle Norden Ungarns in der Struktur der Populationen dem Norden und Westen Europas (s. Schelhorv 1942, Ruszkowskr und Bilinski 1969). Im Gegensatz dazu ist in der Ungarischen Tiefebene - und sogar in ihrem nördlichen Teil (Kompolt : siehe BENEdEK et al., 1971) - die Dominanz der bivoltinen Wildbienen mit mittlerer und langer Flugzeit sehr auffällig (Tabelle 2).

Diese Besonderheit kann durch die zoogeographische Situation des Karpathenbeckens erklärt werden. Denn die meisten der Tetralonia- und Eucera-Arten, die in den Zottelwickenfeldern nachgewiesen werden konnten, erreichen in Ungarn ihre nördliche und westliche Verbreitungsgrenze. Von den Arten mit mittlerer Flugdauer, die in ungarischen Zottelwickenfeldern mit einiger Häufigkeit auftraten (Tabelle 2), wurden nur Eucera longicornis und $E$. interrupta aus Westungarn gemeldet (SсHмпеdeкNEchт 1930), während aus Nord- und Westeuropa in den Zottelwickenfeldern allein E. longicornis in einiger Dichte registriert wurde.

Die Eucera- und Tetralonia-Arten, die auf den ungarischen Zottelwickenfeldern in grosser Häufigkeit gefunden wurden, sind vom pontischen und mediterranem Verbreitungstyp und Ursprung (Sснмперекnechт 1930). Ohne Zweifel haben sie das Karpathenbecken nach der letzten Eiszeit besiedelt. Vermutlich aus klimatischen Gründen traten sie jedoch nur südlich des Ungarischen Mittelgebirges, d.h. in der Ungarischen Tiefebene in grosser Häufigkeit auf. Auch unter den nachgewiesenen bivoltinen und semisozialen Wildbienen mit langer Flugzeit gibt es einige Arten, die ihre Verbreitungsgrenze im Karpathenbecken erreichen (SснміедекNecht 1930). Gleichzeitig wurden die 
restlichen Arten dieser beiden Gruppen im Nordwesten in einer auffällig niedrigeren Häufigkeit gefunden als in der Ungarischen Tiefebene. Daraus kann der Schluss gezogen werden, dass in Südeuropa Zusammensetzung und Dichte der Wildbienen-Populationen von der Zottelwicke ähnlich wie in der Ungarischen Tiefebene sind. Dagegen gleichen die Wildbienen-Populationen auf der Zottelwicke vom nordwestlichen Ungarn in ihrer Zusammensetzung und Dichte denen von Nord- und Westeuropa.

Die Flugperioden der Eucera- und Tetralonia-Arten, die in nennenswerter Häufigkeit nachgewiesen wurden, fallen im wesentlichen mit der Blütezeit der Zottelwicke und der Ungarischen Wicke zusammen (Benedek 1968). Diese Arten sammeln auf den Blüten dieser beiden Wickenarten ohne Mühe (BENEDEK et al. 1973, 1974) und sie bevorzugen die Zottelwicke als Pollenquelle (BENEDEK et al. 1971). Ihr wahrscheinliches Ursprungsgebiet (Mediterraneum, Ponticum) deckt sich mit dem der kultivierten Arten von Winterwicken (Vavilov, 1951).

Aus diesen Feststellungen können eindeutige primäre oder sekundäre Beziehungen zu diesen Wildbienen und den Winterwicken geschlossen werden.

Die Zottelwicke wird von einer Reihe von Wildbienen besucht, die auch bei der Bestäubung der Luzerne (Moczár 1961, Benedek 1969a) und des Rotklees (BENEDEK 1970b) eine bedeutende Rolle spielen. Zottelwicke, Luzerne und Rotklee bieten für diese Wildbienen in aufeinander folgenden Perioden ihrer Flugaktivität die Nahrungsgrundlage. Die drei erwähnten Futterleguminosen sind von ähnlicher oder sogar noch grösserer Bedeutung als Nahrungsgrundlage als andere potentiell geeignete Papilionaceen, meist Unkräuter (BENEDEK 1969b, 1972). Das bedeutet eine wesentliche Beziehung zwischen den bestäubenden Wildbienen bei Zottelwicke, Luzerne und Rotklee. Diese Kulturen sind ihrerseits von entscheidendem Einfluss auf die Dichte ihrer bestäubenden Wildbienen-Populationen. Ohne Zweifel hat die Kultur dieser Pflanzen deutlich zur Ausbreitung einiger der nachgewiesenen Arten in nördlicher Richtung und zur Erhöhung ihrer Populationsdichte beigetragen.

Eingegangen im Juni 1974.

Reçu pour publication en juin 1974.

\section{DANKSAGUNG}

Der Autor dankt den Herren J. Komlodi, Tarhos, Mr. L. Bànk, Mikepéres, Mr. E. Wilhelm, Kismegyer, Mr. E. Gaal, Kenderes und Mr. J. Prenner, Tanakajd für ihre Mitarbeit bei den Feldversuchen.

Für die Unterstützung bei den Bestandsaufnahmen und für die Beistellung der Arbeitsmittel während einer Untersuchung über die Bestäubung der Luzerne und verwandter P flanzen wird der Abteilung P flanzenschutz im Ministerium für Landwirtschaft und Ernährung (Budapest) gedankt. 


\section{RÉSUMÉ}

Il n'existe aucune information sur la densité et l'origine des populations d'abeilles sauvages pollinisatrices de la vesce velue. C'est pour cette raison que nous avons mené une étude approfondie dans des champs de vesce de la Grande Plaine Hongroise (comitats de Békés, Hadju et Szolnok) et des régions nord-ouest de la Hongrie (comitats de Györ et de Vas) entre 1968 et 1971 (fig. 1, tabl. 1).

La densité et la composition spécifique des populations d'abeilles sauvages ont été mesurées par la méthode de l'auteur, dite c méthode des bandes 》(BENEDEK, 1970 a). Au total on a étudié 120 bandes et récolté 5095 abeilles sauvages. On a analysé la densité $D$ et la constance d'apparition $C$ des types de vol et des espèces d'abeilles sauvages.

La densité d'abeilles sauvages était de 300-5 600 par hectare (fig. 2), avec une valeur moyenne de $2117 \pm 121$. Mais la moyenne varie grandement selon les comitats (fig. 3 ) et les années (fig. 4).

On a enregistré dans les champs de vesce en fleurs 65 espèces d'abeilles sauvages représentant 5 types de période de vol (groupes de vol) (fig. 2). On n'a noté que quelques espèces de printemps et d'été à courte période de vol. On n'a pu les observer avec une faible densité que dans quelques champs de la Grande Plaine Hongroise. Par contre les espèces qui ont une période de vol moyenne se rencontraient chez un grand nombre d'espèces. Elles étaient abondantes dans la Grande Plaine Hongroise, tandis que leur densité et leur constance étaient plus faibles dans les régions du Nord-Ouest. Le nombre d'espèces rencontrées d'abeilles bivoltines et semisociales à longue période de vol dépassait celui des autres types de vol. Leur densité, néanmoins, variait considérablement d'un comitat à un autre. On a montré l'existence d'un certain nombre d'espèces eusociales à reproduction continuelle et à longue période de vol.

On a pu établir de nettes différences entre la Grande Plaine Hongroise et les régions nordouest en ce qui concerne la proportion des types de vol et la densité des espèces correspondantes. En raison de la forte prédominance de Bombus, la structure des populations des régions fraîches et humides du nord-ouest est semblable à celle d'Europe septentrionale et occidentale. La dominance de certaines espèces d'Eucera et de Tetralonia dans la Grande Plaine Hongroise est par contre frappante. Ce fait particulier peut s'expliquer par la situation zoogéographique du Bassin des Carpathes. De fait la plupart des espèces d'Eucera et de Tetralonia rencontrées dans les champs de vesce atteignent la limite septentrionale et occidentale de leur aire de répartition en Hongrie. On peut donc conclure que la composition et la densité des populations d'abeilles sauvages pollinisatrices de la vesce velue des régions nord-ouest du pays représentent celles d'Europe septentrionale et occidentale. En contre-partie la composition et la densité des populations d'abeilles sauvages pollinisatrices de la vesce velue en Europe méridionale est semblable à celle de la Grande Plaine Hongroise.

La période de vol des espèces d'Eucera et de Tetralonia rencontrées avec une forte densité coïncide en majeure partie avec la période de floraison de la vesce velue et de la vesce hongroise. Ces abeilles exploitent sans effort les fleurs de vesce velue et de vesce hongroise et préfèrent les vesces d'hiver comme source pollinifère. Leur aire possible d'origine dépasse celle des variétés cultivées de vesce d'hiver. Ces conditions déterminent une relation primaire ou secondaire bien définie entre ces abeilles sauvages et les vesces d'hiver.

Les champs de vesce velue sont visités par plusieurs espèces d'abeilles sauvages qui jouent un rôle important dans la pollinisation de la luzerne et du trè fle rouge. Ces cultures peuvent donc avoir une in fluence mutuelle précise sur la densité de leurs populations d'abeilles sauvages pollinisatrices. 


\section{LITERATUR}

Benedek P., 1968. The flight period of wild bees - Hymenoptera, Apoidea — pollinating lucerne, and its plant protection aspects. Acta Phytopath. Acad. Sci. Hung., 3, 59-71.

BENEDEK P., 1969 a. Examinations on lucerne pollinating wild bees - Hym., Apoidea - in the Hungarian Great Plain. Fol. Ent. Hung., 22, 101-115.

BENEDEK P., $1969 \mathrm{~b}$. Flower visiting habits of lucerne pollinating wild bees and the increasing of wild bee populations by providing unbroken feeding possibilities. Z. ang. Ent., 63, 186-199.

BENEDEK P., 1970 a. Effects of plot size, stand density and state of flowering on wild bees pollinating lucerne. Acta Agron. Hung., 19, 283-292.

BENEDEK P., 1970 b. Investigations on red clover pollinating wild bees on the Great and Small Plain of Hungary. Növénytermelês, 19, 361-371. In Hungarian with English summary.

BenedeK P., 1972. Possible indirect effect of weed control on population changes of wild bees pollinating lucerne. Acta Phytopath. Acad. Sci. Hung., 7, 267-278.

BenedeK P., BÁnK L., Kombódr J., 1973. Behaviour of wild bees - Hymenoptera : Apoidea on hairy vetch - Vicia villosa Roth. - flowers. Z. ang. Ent., 74, 80-85.

Benedek P., Buglos J., Manninger S., 1971. Observations on wild bees visiting winter vetches. Növénytermelés, 20, 337-346. In Hungarian with English summary.

BenedeK P., Wilhelm E., GaÁl E., Komlódi J., 1974. Observations on apoids - Hymenoptera : Apoidea - visiting Hungarian vetch fields. Növênytermelês, in preparation. In Hungarian with English summary.

Bohart G.-E., 1960. Insect pollination of forage legumes. Bee World, 41, 57-64 and 85-97.

MóczÁr L., 1961. The distribution of wild bees in the lucerne fields of Hungary - Hymenoptera, Apoidea -. Ann. Hist. Nat. Mus. Nat. Hung., 53, 451-461.

Ruszkowski A., Bilinski M., 1969. Trzmiele oblatujace wyke i inne rosliny straczkowe. Pamiet. Pulaw., 36, 281-299.

Schelmorv M., 1942. Blütenbiologische Studien an der Zottelwicke. Pflanzenbau, 18, 311-320.

Schelnors M., 1946. Blütenbiologie und Samenansatz bei Vicia villosa. Züchter, 17, 22-24.

Schmiedeknech' O., 1933. Die Hymenopteren Nord- und Mitteleuropas. Gustav Fischer, Jena.

VAviLov N. I., 1951. The origin, variation, immunity and breeding of cultivated plants. Chronica Botanica, 13, 1-364. 